\title{
Role of Cetuximab Re-introduction and Re-challenge in Later Lines of Treatment in Metastatic Colorectal Cancer: A Case Series
}

\author{
Amrou Shaaban ${ }^{1,2^{*}}$, Asit Mohanty ${ }^{1}$, Yousef Abo Elseud ${ }^{1}$, Jasem Albarrak ${ }^{1}$ \\ ${ }^{1}$ Kuwait Cancer Control Centre, Shuwaikh, Kuwait \\ ${ }^{2}$ Clinical Oncology Department, Faculty of Medicine, Minia University, Egypt.
}

*Corresponding Author: Amrou Shaaban, Email: genzo_2010@yahoo.com

\section{Abstract}

Colorectal cancer (CRC) is the third-largest cancer in the world and has the second-highest mortality rate, characterized by poor prognosis and high metastasis. The early symptoms of CRC are mostly inconspicuous and it is usually diagnosed in the advanced stages. Nearly half of the patients diagnosed with metastatic colorectal cancer $(\mathrm{mCRC})$ are inoperable and have high chances of recurrence and metastasis. Combination therapy with cetuximab and chemotherapy is considered the first-line treatment for mCRC. In patients with Kirsten RAt Sarcoma wild-type $\mathrm{mCRC}$, re-challenge and maintenance therapy with cetuximab was found to be beneficial. However, the disease progression is inevitable. This paper discusses three different cases of metastatic adenocarcinoma in the colon and rectum treated with the re-introduction and re-challenge of cetuximab in combination with chemotherapy. The treatment was found to be effective in treating $\mathrm{mCRC}$ in later lines of therapy where treatment options are scarce.

Keywords

Cetuximab; Chemotherapy; Metastatic disease, Colorectal cancer; Re-challenge; Re-introduction.

\section{INTRODUCTION}

Colorectal cancer (CRC) is the third most common cancer in the world and has the second-highest mortality rate. The five-year survival rate for localized CRC and regionalized CRC is $90 \%$ and $70 \%$, respectively, whereas for patients with metastatic CRC (mCRC) it is only $13 \%{ }^{[1,2]}$. In recent developments, targeted biologic therapies such as antiepidermal growth factor receptors (anti-EGFR; first-line treatment), anti-vascular endothelial growth factor (anti-VEGF; second-line treatment), and monoclonal antibodies are known to be effective in the treatment of mCRC. The targeted therapies are effective in the management of $\mathrm{mCRC}$ in combination with chemotherapy due to lesser toxicities and better outcomes as compared with traditional chemotherapy in the third line of treatment ${ }^{[2]}$. Also, previous studies indicated 
that combining chemotherapy with anti-EGFR therapy has a positive impact on overall survival (OS) and progression free survival (PFS) ${ }^{[1,3]}$. However, these benefits were only specific to patients with wild-type rat sarcoma (RAS) genes. It has been observed that continuous treatment with anti-EGFR therapy can increase the risk of making tumor cells resistant ${ }^{[4]}$. At baseline, the wild-type RAS CRC can acquire resistance to anti-EGFR treatment via a RAS status 'shift' to mutated status to escape the drug's effects ${ }^{[5]}$. Thus, continuous therapy despite being responsive is not considered advantageous compared to 're-introduction' and 're-challenging' strategies ${ }^{[6]}$. Re-introduction refers to the interruption of therapy in patients without any evidence of tumor resistance and its subsequent reintroduction in another line of treatment after a gap. 'Re-challenging' is based upon the re-initiation of the same drug or treatment regimen to which the tumor has already been proven to be resistant. During the treatment gap, the patient may not be given any treatment, termed 'treatment holiday'; or may receive an intervening therapy other than anti-EGFR therapy ${ }^{[7]}$. Monitoring of the plasma levels of RAS-mutant circulating tumor DNA has shown that gaps in anti-EGFR therapy may help in restoring the sensitivity of tumors to biologic therapies including cetuximab ${ }^{[7]}$. Also, an analysis showed that KRAS or EGFR relative mutant alleles can decay with a half-life of nearly 4.4 months. Based on this model, it was concluded that patients with increased time-intervals between the first treatment and re-challenging had a higher overall response rate ${ }^{[8]}$. Thus, both re-introduction and re-challenging with anti-EGFR therapy can be deemed as lucrative strategies in the management of mCRC. The other benefits of interrupted treatment with anti-EGFR therapies include less cumulative toxic effects (in re-introduction) and maximum management of adverse events (in re-challenging) which contribute to increasing OS and improving quality of life ${ }^{[9]}$.

Finally, the reduction in cost implications is another positive aspect of these strategies ${ }^{[7]}$. Thus, re-treatment (re-introduction or re-challenging) with anti-EGFR can be considered as an important treatment option in mCRC patients for improved outcomes ${ }^{[9]}$. Here, we report three cases of re-introduction and re-challenge of cetuximab, an anti-EGFR therapy, in combination with chemotherapy, which appeared to be effective in the treatment of $\mathrm{mCRC}$ in later lines of therapy where treatment options are scarce. All the patients have given informed consent for the publication of this case series.

\section{CASE REPORTS}

\section{Case 1}

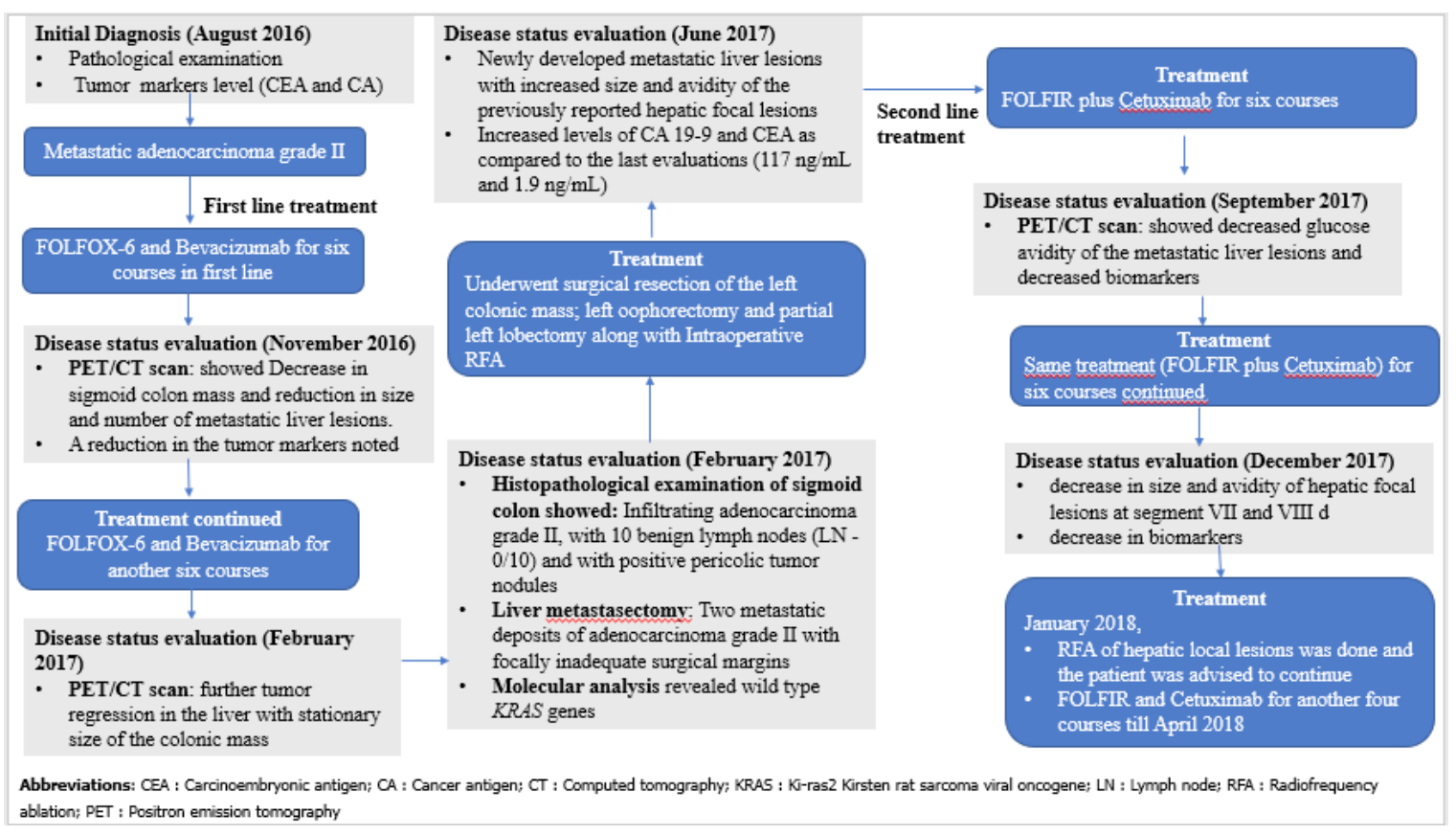

Figure 1. Treatment of Case 1 in Egypt 
A 41-year-old female with previous good health and no family history of cancer presented with altered bowel habits, rectal bleeding, and lower abdominal pain. In August 2016, a colonoscopy revealed a cauliflower mass around $20 \mathrm{~cm}$ from the anal verge. A pathological examination revealed an infiltrating metastatic grade II adenocarcinoma. Laboratory tests indicated elevated levels of tumor markers, carbohydrate antigen 19-9 (CA 199: $4462 \mathrm{ng} / \mathrm{mL}$ ), and carcinogenic embryonic antigen (CEA: $181 \mathrm{ng} / \mathrm{mL}$ ). However, all other tests, such as liver function test, renal function test, and complete blood count, were normal except for a low hemoglobin level of $9 \mathrm{~g} /$ dL. The initial treatment was completed in Egypt where the patient received first- and second-line treatments with FOLFOX-6 plus bevacizumab and FOLFIRI plus cetuximab, respectively. The complete details of the treatment in Egypt are tabulated in Figure 1.

In April 2018, the patient was referred to the Medical Oncology Department in Kuwait Cancer Control Centre (KCCC). A new positron emission tomography (PET)/computed tomography (CT) scan displayed no active disease but indicated ablated lesions. The patient was advised to start maintenance therapy with capecitabine for four courses, till July 2018. She was monitored closely for disease status by CT scans, PET/CT, and tumor markers every three months.

In August 2018, a CT scan exhibited three new hepatic lesions in segment VII, following which FOLFIRI plus cetuximab was re-introduced as the third line of treatment. A CT scan in November 2018 indicated disease regression in the form of lesions in segments III, VII, and VIII which had the same appearance as post radiofrequency ablation (RFA) changes. Also, the previously detected three lesions in segment VII became smaller in size. The tumor biomarker levels were also found to be normal. The patient was advised to continue the same treatment regimen for another six cycles. In February 2019, the CT scan demonstrated no appreciable interval changes in the post-RFA focal lesions at segments III, VII, and VIII; while the previously developed two lesions in segment IVa and IVb were almost stable and presented necrotic changes indicating a response to treatment. Besides this, no new focal lesions were observed, and the tumor markers continued to be within a normal range.

As treatment with FOLFIRI plus cetuximab was successful in controlling the disease and was well tolerated by the patient, it was decided to continue her on the same treatment. The last follow-up was done in July 2020.

\section{Case 2}

A 61-year-old male presented with dyslipidemia, hypertension, and ischemic heart disease for which he was on candesartan and aspirin. He had no family history of malignancies. He was referred to our hospital in November 2014 with a history of constipation for two months and a weight loss of $7 \mathrm{~kg}$. A CT scan of the chest, abdomen, and pelvis done on November 13, 2014, revealed circumferential thickening of the sigmoid wall, and enlarged mesenteric para-aortic mediastinal lymph nodes (Figure 2 [a] and [b]). The magnetic resonance imaging (MRI) of the pelvis done on November 16, 2014, exhibited diffused circumferential thickening of the sigmoid colon. A colonoscopy done on November 17, 2014, indicated a mass lesion at $10 \mathrm{~cm}$ from the anal verge, while the rest of the colon examination was normal. A biopsy confirmed rectal adenocarcinoma in November 2014. Molecular analysis confirmed wild-type RAS and BRAF-MSS genes. The initial diagnosis was done in Germany and the patient was treated there for three years (November 2014 to November 2017). He received four lines of treatment starting with chemoradiation of the pelvis with FOLFOX-4, followed by FOLFIRI plus cetuximab plus bisphosphonate. Nine courses of chemotherapy with FOLFIRI plus cetuximab plus bone modifying agent were resumed from December 2016 to May 2017. The details of the treatment are presented in Figure 3. 


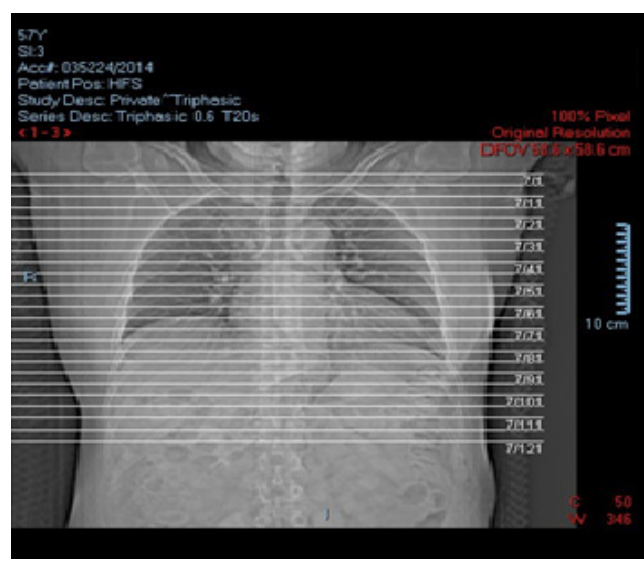

a) CT scan of Chest

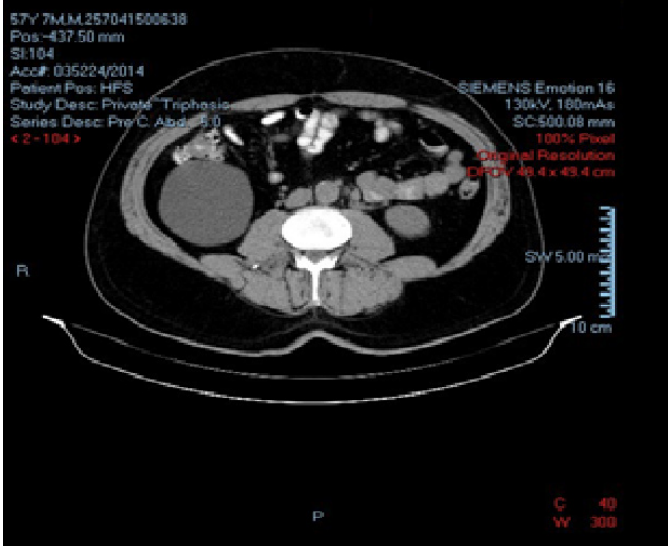

b) CT scan of Abdomen

Figure 2. CT scan of Chest and Pelvis for Case 2 at the time of diagnosis: (November 13th, 2014)

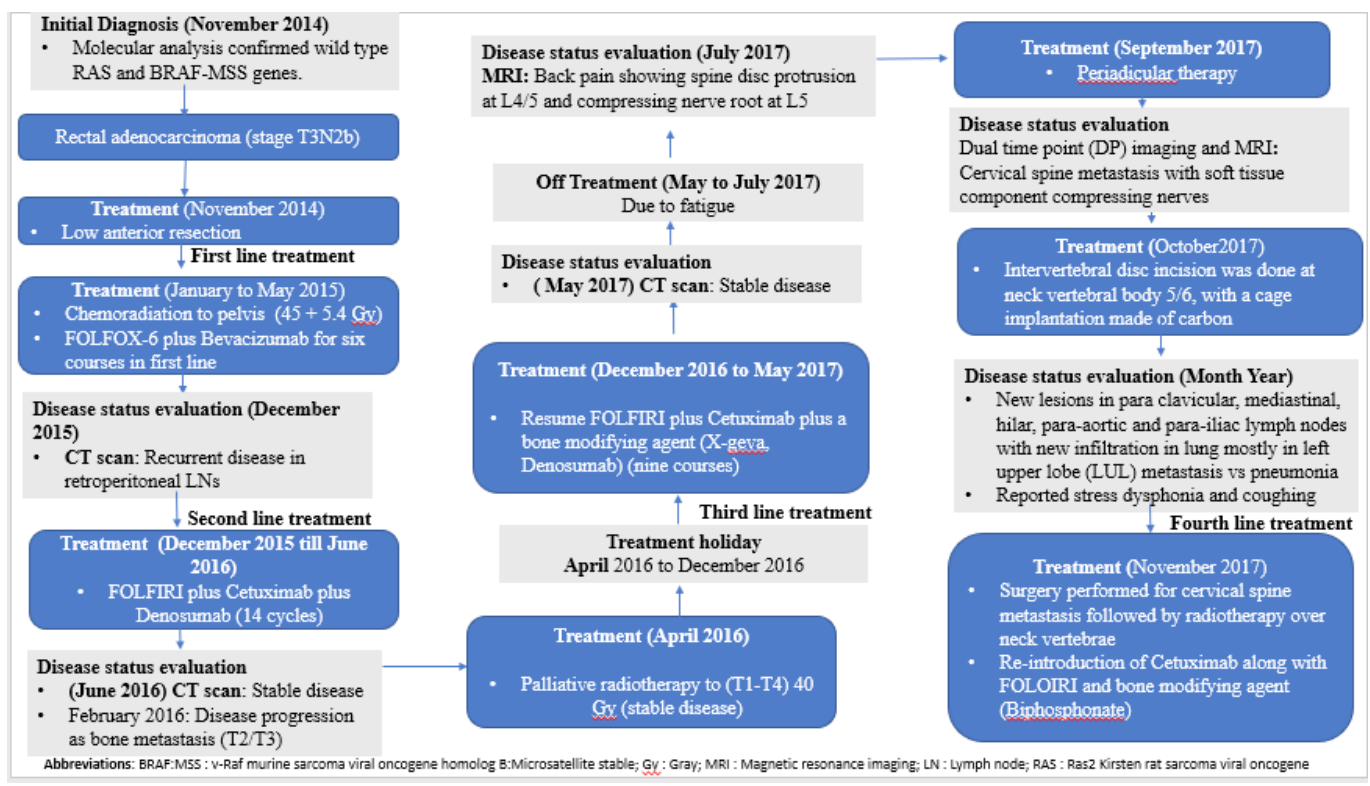

Figure 3. Treatment of Case 2 in Germany

The patient returned to Kuwait in April 2018, and the same treatment with FOLFIRI plus cetuximab along with bisphosphonate was resumed for nine cycles. A CT scan evaluation in June 2018 indicated stable disease, but an MRI of the spine revealed progression of spinal metastasis with a newly developed lesion at L5. This was not indicated for spinal surgery and conservative measures were recommended. The patient resumed treatment with FOLFIRI plus cetuximab. At the end of July 2018, a CT scan of the chest, abdomen, and pelvis indicated stable disease (Figure 4 ([a], $[\mathbf{b}]$, and $[\mathbf{c}]$ ), and the same treatment protocol was continued along with co-management of pain by the palliative care team. In October 2018, the last cycle of chemotherapy was given, and the patient tolerated it well. He then traveled to the United States of America (USA) to seek a second opinion regarding his current metastatic bone disease, at the Dana Farber Cancer Institute. In November 2018, the patient experienced progressive pain in the abdomen and left upper extremity which was explained to be caused by his metastatic cancer, and was managed with a combination therapy of gabapentin, nortriptylin, MS contin, and morphine sulfate immediate release (MSIR). The patient also received steroid and lidocaine injection at C6-C7 (neck and left arm); this was effective in controlling pain. Further, colonoscopy and biopsy of the anastomotic area indicated only colitis with no evidence of malignancy. The patient was advised to begin the second line of treatment with FOLIFOX and bevacizumab along with pegfilgrastim support. The patient completed nine cycles in the U.S. and 
further evaluation of chest, abdomen, and pelvis by a CT scan indicated stable disease and neuropathy related to cold weather.

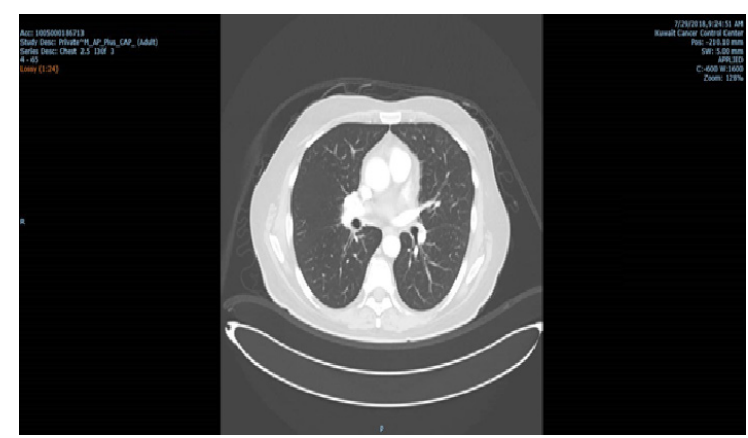

Figure 4 (a)

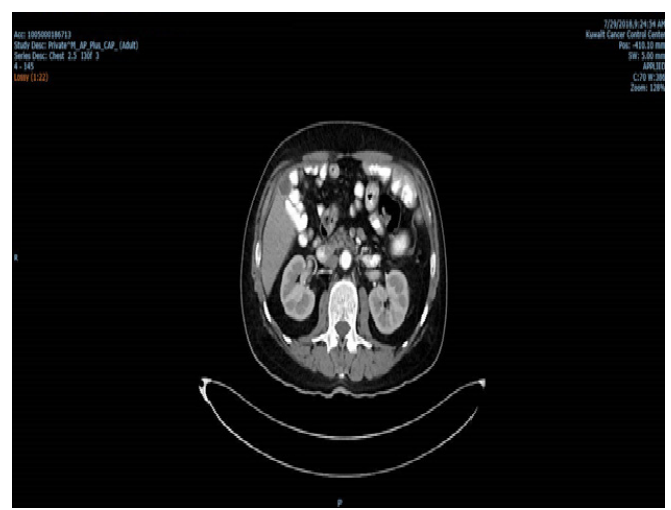

Figure 4 (b)

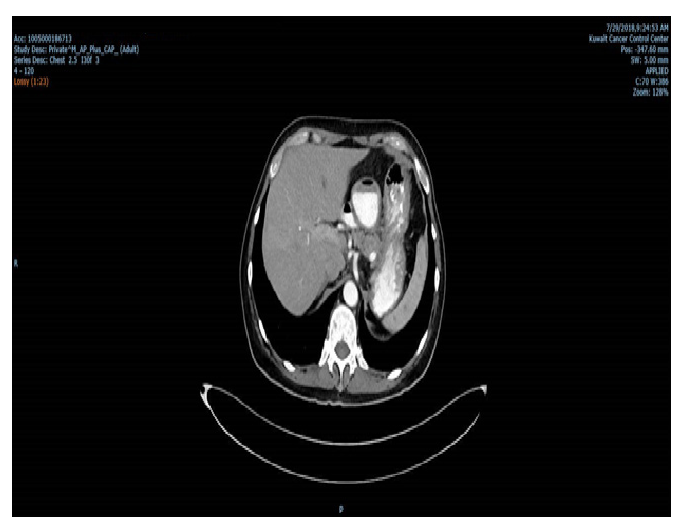

Figure 4 (c)

Figure 4. CT scan images of Case 2 were done on July 29th, 2018. a) CT of the chest, b) CT of Abdomen, c) CT of Pelvis.

In May 2019, the patient returned to KCCC in Kuwait, with Eastern Cooperative Oncology Group (ECOG) PS-0 but had left upper limb neuropathic pain. An MRI of the spine displayed a patchy marrow infiltrative process seen at multiple levels of the cervical and upper dorsal vertebrae, most prominent at the D4 vertebral body, which was related to his metastatic disease. The patient was advised to continue treatment with FOLFOX plus bevacizumab for 12 cycles till June 2019. The disease evaluation by MRI of the spine in July 2019 disclosed slightly more prominent multiple vertebral body metastasis with associated soft tissue component bordering the 
spinal cord at the level of C6, causing significant spinal cord compression at the level of D4 (previously irradiated in Germany in April 2016). A CT scan revealed progressive multiple suspicious vertebral body lesions with related pre-vertebral soft tissue components and lesions. Also, an 18F-Sodium Fluoride (NaF) - PET/CT scan indicated subsequent left vocal cord paralysis and osteoblastic activity in vertebrae C6, C7, D1, D2, D4, and L5, respectively.

As there was disease progression on the current chemotherapy line (FOLFOX-6 plus bevacizumab), the third line of therapy with regorafenib was planned, along with radiotherapy consultation to check if there were minimal chances for re-irradiating the D4 metastatic lesion. For pain management, the patient was referred outside KCCC in July 2019. The patient was last followed up on July 19, 2019, wherein his active chemotherapy treatment was on hold due to his poor general health.

\section{Case 3}

A 59-year-old male with no previous family history of cancer was diagnosed with metastatic sigmoid colon adenocarcinoma with KRAS wild-type genes in May 2012. The comorbidities included arterial hypertension and a history of pulmonary embolism. The initial treatment of the patient was conducted in the UK, where the patient received the first line of treatment with FOLFOX plus bevacizumab. The treatment history is presented in Figure 5.

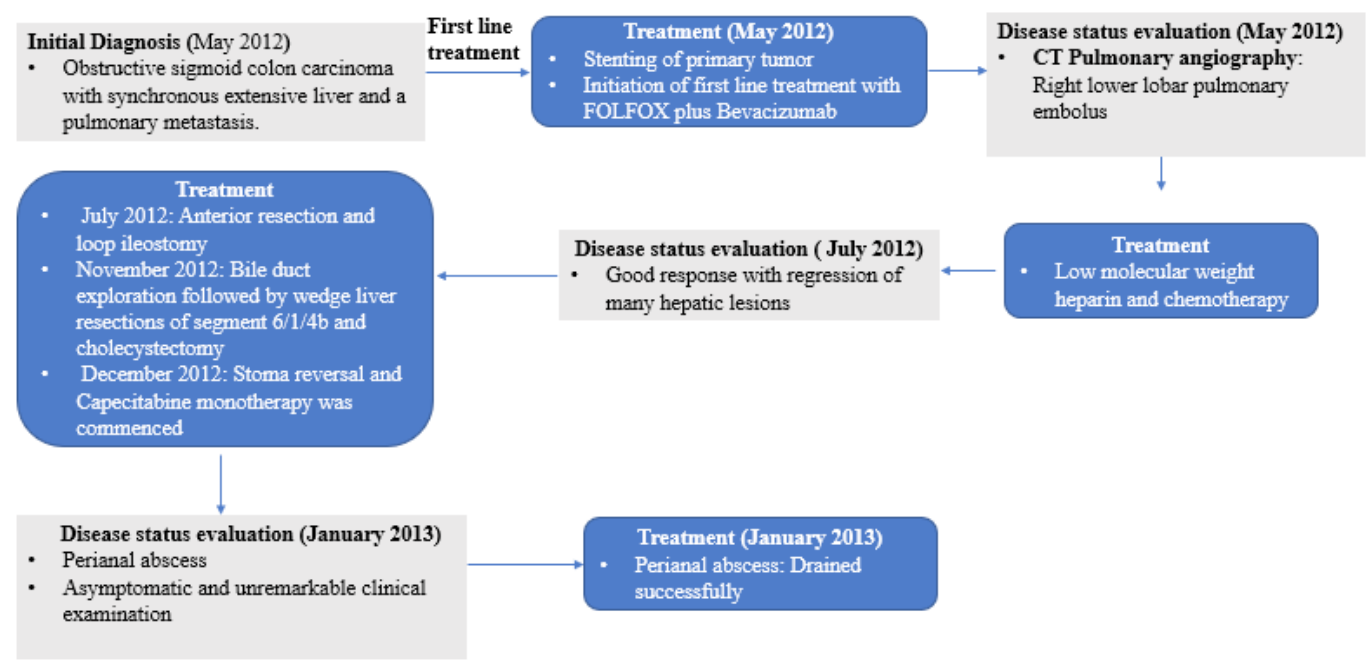

Figure 5. Treatment of Case 3 in the UK

The patient returned from the UK to the Oncology Department in KCCC, Kuwait. In May 2013, an MRI of the liver exhibited postoperative changes in segment 6 of the liver and multiple suspicious focal lesions in the right hepatic lobe, mainly at segments 6,5 , and 4a. A PET/CT scan revealed hypermetabolic pulmonary lesion at the lateral segment of the left lower lobe and multiple follicular dendritic cell (FDC) avid hepatic focal lesions in segments 6,7, and 8 (Figure 6 [a], [b]). The tumor marker CEA levels were normal. The patient was advised to initiate treatment with anti-EGFR, but he refused as he was apprehensive about his facial looks. He had persistent polyneuropathy. So, the patient was suggested a second line of treatment with FOLFIRI plus bevacizumab after repeat port insertion. Later, in June 2013, another 18F-fluorodeoxyglucose (FDG) PET/CT scan revealed avid lung, liver, and peritoneal metastasis, and the patient was advised to initiate treatment with FOLFIRI plus bevacizumab. For disease evaluation, a PET scan was conducted in August 2013, which indicated an excellent response in all lesions, and systemic therapy was continued. In the same month, a left lower lung lobectomy revealed mCRC, and the patient was suggested to continue FOLFIRI plus bevacizumab, till November 2013, though bevacizumab was omitted in the final cycles. In December 2013, the MRI of the liver revealed three 
unchanged liver lesions after treatment. Hence, the patient underwent liver resection and intra-operative ablation. The histology results after surgery revealed metastatic adenocarcinoma with positive margins at some points. In February 2014, the patient was advised to initiate FOLFOX plus bevacizumab as the third line of treatment. In July 2014, a new PET/CT confirmed FDG avid nodule in the left lower pulmonary lobe, and the fourth line of therapy with FOLFIRI plus cetuximab was started. Left lower lobectomy was done. In September 2014, a PET/ CT scan indicated improvement in disease, and thus, systemic therapy was continued. Another PET/CT scan in January 2015, indicated stable disease after completion of cetuximab-based therapy, and the patient was advised to start maintenance therapy with capecitabine. Another evaluation of the patient done in April 2015 with a PET/CT scan disclosed the presence of a new right middle lobe lung nodule (suggestive of metastasis). In May 2015, the presence of right middle lobe nodules was confirmed by another CT scan. In November 2015, the patient traveled to the UK for a second opinion. There, he was advised to continue with capecitabine as a PET/CT scan showed no signs of disease recurrence. New CT and PET/CT scans were conducted in November and December 2015, respectively, which showed no evidence of disease recurrence. Also, CEA levels were found to be normal.

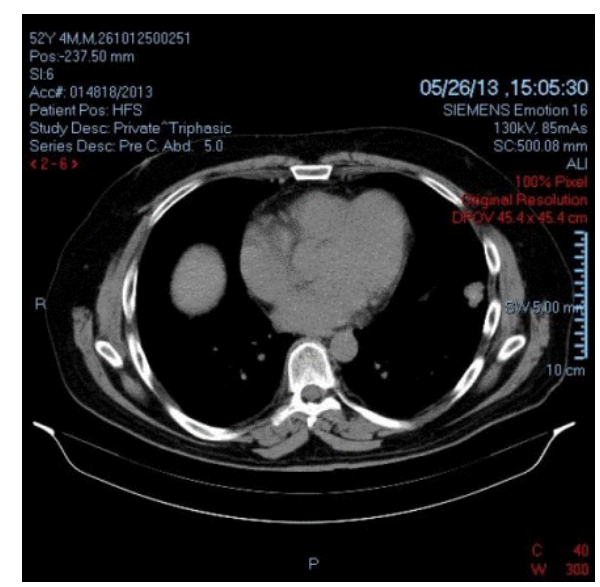

Figure 6 (a)

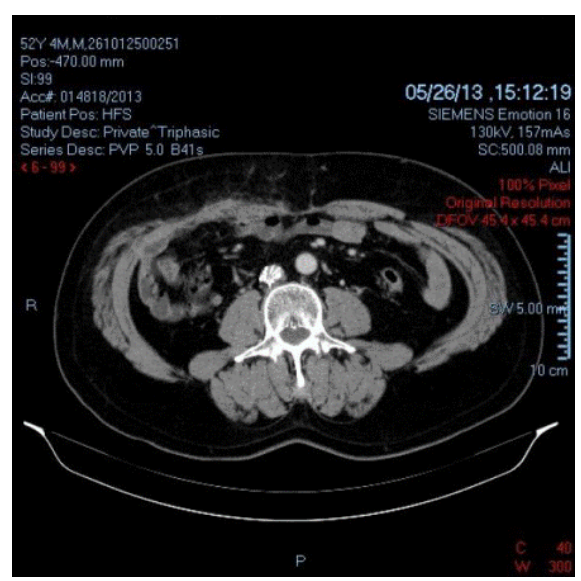

Figure 6 (b)

Figure 6. CT scan images of Case 3 done on May 26th, 2013. a) CT of a hypermetabolic pulmonary lesion at the lateral segment of left lower lobe, b) CT of FDC avid hepatic focal lesions in segment 6, 7, and 8

On returning to Kuwait, repeated scans were conducted in May 2016, which suggested a new hypermetabolic lesion in the right liver lobe, uptake at hepatic flexure, and non-FDG avid right middle lobe pulmonary nodule. CT scans confirmed the newly developed hepatic focal lesion. The patient was advised to undergo an MRI of the liver to confirm the disease recurrence, but he wanted to get a second opinion regarding disease recurrence and thus 
traveled to the UK again. The patient was last seen in May 2016 with ECOG PS-0.

Back in the UK, the patient was confirmed to have a progression of hepatic metastasis and was advised to initiate the fifth line of treatment with FOLOFIRI plus panitumumab in a total of eleven courses. Later in July 2016, the evaluation suggested a good response to chemotherapy with remaining metastasis measuring $1 \mathrm{~cm}$ that was completely ablated with microwaves. In October 2016, a PET scan confirmed complete remission of the disease, and the patient was advised to undergo another three courses of FOLOFIRI plus bevacizumab in the sixth line of treatment, followed by maintenance therapy with a low dose of capecitabine. The maintenance therapy with capecitabine was continued till January 2017. Later, in April 2017, the patient returned to Kuwait, and the disease progression was confirmed with a PET scan and increased CEA levels. The patient was suggested palliative chemotherapy. However, he wanted to seek another opinion and again traveled to the UK, with ECOG PS 1, in June 2017.

In the UK, the patient was reported to have progressive hepatic and pulmonary metastasis and underwent hepatic and pulmonary resection. The postoperative CT scan still displayed two liver lesions, a $14 \mathrm{~mm}$ lesion in segment 2 and an additional $11 \mathrm{~mm}$ liver lesion close to the previous surgery. A re-challenge with FOLFOX plus cetuximab in the seventh line of treatment for twelve cycles was done. The patient later showed a complete metabolic response in PET, and no evidence of residual disease was observed in a CT scan (Figure 7).The patient returned to Kuwait and new PET/CT and CT scans in February 2019 confirmed disease progression with the emergence of new hypermetabolic nodal, pulmonary, and hepatic lesions favoring metastatic disease progression. His ECOG PS was 1.

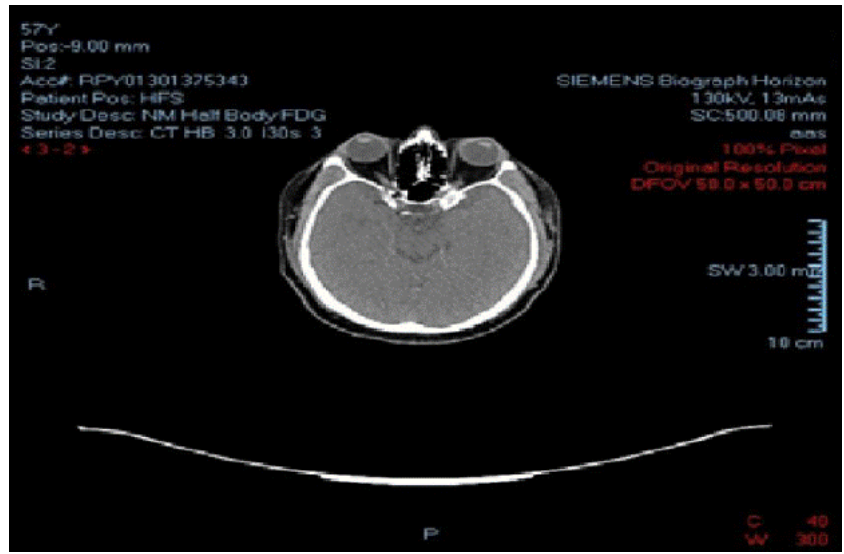

Figure 7 PET scan for Case 3 (June 21st, 2018)

The treatment plan was to re-challenge with palliative chemotherapy and continuation of FOLOFIRI plus cetuximab, as it was still working for the patient. He commenced 2 cycles in Kuwait, then traveled to the UK where further 4 cycles were continued at the Royal Marsden Hospital (RMH) through May 2019, with some reduction in the size of the liver lesions. Further, 6 cycles of the same treatment were continued till August 2019, with an evaluation suggesting progressive disease in the liver and lung. There was also thrombosis in a branch of the portal vein and he started low molecular weight anticoagulation with INNOHEP ${ }^{\circledR}$. The UK team recommended him to change treatment to FOLFOX, as he had shown a response to it previously. He was advised to cancel ablation at this stage until systemic disease control was achieved. The patient returned to Kuwait in October 2019. The hematology team reviewed him and changed his medication to the oral anticoagulant, rivaroxaban. On October 24, 2019, the patient experienced melena with blood loss. He received a blood transfusion. Clinically, the patient had jaundice and abdominal ultrasound suggested no biliary dilation. Magnetic resonance cholangiopancreatography (MRCP) indicated biliary obstruction in the left hepatic duct. In early 
November 2019, biliary drainage was done through successful percutaneous transhepatic biliary dilatation (PTD). Unfortunately, his general condition was complicated with biliary sepsis and pneumonia. He was admitted to the ICU. However, his condition deteriorated, and the patient died in December 2019.

\section{DISCUSSION}

Here we report two cases of 're-introduction' and one case of 're-challenging' mCRC patients with a combination of cetuximab and chemotherapy. The response to re-treatment was evident from the clinical improvement in patients, disease stability, and a drop in the tumor marker levels.In the first case, cetuximab along with FOLFIRI was initiated as the second line of treatment and later as the third line after maintenance therapy. The re-introduction led to the regression of the disease as seen in the CT scan and tumor markers being in the normal range. In the second case, cetuximab was introduced as the third line of treatment and then stopped due to fatigue and re-introduced as the fourth line. Thus, with re-introduction, the quality of life of both the patients was improved for a significant period.

A study assessed the re-introduction of cetuximab in five patients with $\mathrm{mCRC}$ who received it as first-line therapy. The drug was then discontinued without any disease progression. On re-introduction after a gap (median time interval 14.1 months), cetuximab resulted in improved OS and no severe toxicity was reported. Out of five patients, one displayed partial remission (OS: 45 months), two had stabilized disease (OS: 30.2 months and 50.5 months), while two had progressive disease (OS: 35 months and 43 months) [10]. Another recent case-control study assessed the effectiveness of re-introducing cetuximab-chemotherapy $(n=22)$ over chemotherapy alone $(\mathrm{n}=22)$ after a break, in wild-type KRAS mCRC patients treated with cetuximab-chemotherapy as first/secondline treatment with the best objective response rates. The study results indicated re-introduction of cetuximabchemotherapy to be more effective than chemotherapy alone for median OS (18.6 months; $95 \%$ CI: 11.1-27.1 vs. 11 months; $95 \%$ CI: $4.5-17.1)$ and 1 -yr PFS (36.4\% vs. $22.7 \%)$, respectively ${ }^{[1]}$. However, there is a need to conduct more studies to identify the right population that can benefit from re-introduction.

In the third case, the patient with wild-type KRAS mCRC was given cetuximab initially as the fourth line of treatment and later re-challenged in the seventh line due to disease progression from an intervening therapy and maintenance therapy in the sixth line of treatment. A CT scan indicated the effectiveness of re-challenging with cetuximab as no evidence of residual disease was found.

The CRICKET trial was aimed at assessing the effectiveness of irinotecan plus cetuximab re-challenging when given as the third line of treatment to mCRC patients with RAS and BRAF wild type, who had initially received the drug as the first line of treatment and then acquired resistance. The study results demonstrated the effectiveness of re-challenging in 25/28 evaluable patients, of which partial response and stable disease was seen in six and nine patients, respectively ${ }^{[12]}$. In a retrospective study, irinotecan plus cetuximab re-challenge exhibited a median PFS of 3.5 months. Further, disease stabilization and partial response were reported in $26.7 \%$ and $13.3 \%$ of patients, respectively ${ }^{[13]}$. In another recent case report, the effectiveness of re-challenge and maintenance therapy with cetuximab-based chemotherapy was demonstrated with significant shrinkage of the metastatic lesions in the lung and liver of a female patient with wild-type KRAS. Her level of tumor biomarkers also dropped ${ }^{[14]}$.

\section{CONCLUSION}

Re-introduction and re-challenge with cetuximab plus chemotherapy can be considered as an effective option for treating mCRC patients with wild-type KRAS and BRAF genes. However, more evidence from randomized controlled trials with large sample sizes is required to confirm the benefit and to find out which populations can 
benefit most from re-treatment with cetuximab.

\section{DISCLOSURE/ACKNOWLEDGEMENT}

The development of this publication was financially supported by Merck Serono Middle East FZ - LTD, Dubai, UAE; an affiliate of Merck KGaA, Darmstadt, Germany through an independent medical writing grant. The views and opinions described in this publication do not necessarily reflect those of the grantor. Medical writing services were provided by Turacoz Healthcare Solutions. The manuscript was further edited by Rupali Bahri, Medcytes, Dubai.

\section{AUTHOR CONTRIBUTIONS}

Shaaban, A. Mohanty, Y. Abo Elseud, and J. Albarrak contributed to the implementation of the study, the analysis of the results, and the writing of the manuscript.

\section{CONFLICT OF INTEREST}

The authors declare no potential conflicts of interest concerning the research, authorship, and/or publication of this study.

\section{REFERENCES}

[1] Salvatore L, Aprile G, Arnoldi E, Aschele C, Carnaghi C, Cosimelli M, et al. Management of metastatic colorectal cancer patients: guidelines of the Italian Medical Oncology Association (AIOM). ESMO Open. 2017;2(1):e000147.

[2] Rawla P, Barsouk A, Hadjinicolaou AV, Barsouk A. Immunotherapies and Targeted Therapies in the Treatment of Metastatic Colorectal Cancer. Med Sci (Basel). 2019;7(8).

[3] Segelov E, Chan D, Shapiro J, Price TJ, Karapetis CS, Tebbutt NC, et al. The role of biological therapy in metastatic colorectal cancer after first-line treatment: a meta-analysis of randomised trials. Br J Cancer. 2014;111(6):1122-31.

[4] Leto SM, Trusolino L. Primary and acquired resistance to EGFR-targeted therapies in colorectal cancer: impact on future treatment strategies. J Mol Med (Berl). 2014;92(7):709-22.

[5] Goldberg RM, Montagut C, Wainberg ZA, Ronga P, Audhuy F, Taieb J, et al. Optimising the use of cetuximab in the continuum of care for patients with metastatic colorectal cancer. ESMO Open. 2018;3(4):e000353.

[6] Santini D, Vincenzi B, Addeo R, Garufi C, Masi G, Scartozzi M, et al. Cetuximab rechallenge in metastatic colorectal cancer patients: how to come away from acquired resistance? Ann Oncol. 2017;28(11):2906.

[7] Tonini G, Imperatori M, Vincenzi B, Frezza AM, Santini D. Rechallenge therapy and treatment holiday: different strategies in management of metastatic colorectal cancer. Journal of experimental \& clinical cancer research. 2013;32(1):92.

[8] Parseghian CM, Loree JM, Morris VK, Liu X, Clifton KK, Napolitano S, et al. AntiEGFR-resistant clones decay exponentially after progression: implications for anti-EGFR rechallenge. Ann Oncol. 2019;30(2):243-9.

[9] Van Cutsem E, Cervantes A, Adam R, Sobrero A, Van Krieken JH, Aderka D, et al. ESMO consensus guidelines for the management of patients with metastatic colorectal cancer. Ann Oncol. 2016;27(8):1386422.

[10] Kaechele V, von Wichert G, Adler G, Seufferlein T. Response to reintroduction of cetuximab in 5 patients with advanced, chemotherapy-resistant, colorectal cancer without progressive disease following first-line therapy with a cetuximab-containing regimen. Oncology. 2008;74(3-4):123-6.

[11] Ho CS, Cheng AC, Li L, Ho WM, Hui EP, To KF, et al. A pilot case-control study of second or third-line treatment with cetuximab-containing chemotherapy (cetux-chemo) in patients (pts) with metastatic colorectal 
cancer (mCRC) who were previously treated with cetuxchemo. Annals of Oncology. 2018;29.

[12] Cremolini C, Rossini D, Dell'Aquila E, Lonardi S, Conca E, Del Re M, et al. Rechallenge for Patients With RAS and BRAF Wild-Type Metastatic Colorectal Cancer With Acquired Resistance to First-line Cetuximab and Irinotecan: A Phase 2 Single-Arm Clinical Trial. JAMA Oncol. 2019;5(3):343-50.

[13] Nogueira A, Rodrigues J, Jacinto P, Ribeiro J, Bonito N, Marques M, et al. P-167 Cetuximab rechallenge in metastatic colorectal cancer patients. Annals of Oncology. 2016;27.

[14] Ma J, Yang QL, Ling Y. Rechallenge and maintenance therapy using cetuximab and chemotherapy administered to a patient with metastatic colorectal cancer. BMC Cancer. 2017;17(1):132. 